\title{
Clarificación de aceite de cocina usado y decoloración de aceite rojo de palma con el uso de ozono, carbón activado y peróxido de hidrógeno
}

\section{(Clarification of used cooking oil and discoloration of red palm oil with the use of ozone, activated carbon and hydrogen peroxide)}

\author{
Tania Parra ${ }^{1}$, Freddy Marín ${ }^{2}$, Gonzalo Jácome ${ }^{1}$, Marco Sinche ${ }^{1}$
}

\begin{abstract}
Resumen:
Se evaluaron procesos alternativos para la clarificación de aceite de cocina usado (ACU) y la decoloración de aceite rojo de palma (ARP). La clarificación del ACU se desarrolló en dos etapas, una remoción de sedimentos y una decoloración. En la primera etapa se probaron tres métodos: calentamiento, lavado con salmuera, sedimentación y filtración; solamente sedimentación; y lavado con salmuera, sedimentación y filtración. El tercer método permitió la mayor eliminación de impurezas. Para la segunda etapa también se probaron tres métodos: adsorción con carbón activado (CA); ozonización y aplicación de peróxido de hidrógeno. El mejor método fue la ozonización con una dosis de $0,1946 \mathrm{~mol} \mathrm{O}_{3} / \mathrm{L}$ y una temperatura postratamiento de $60 \stackrel{\circ}{\circ}$; se alcanzó un porcentaje de decoloración de $24,4 \%$. Para el ARP, el mejor tratamiento de decoloración fue la adsorción con carbón activado. La relación de aceite: CA que generó los mejores resultados fue 25:1; se logró una disminución de color del $90,48 \%$. El análisis de los parámetros de calidad medidos en los aceites tratados (índice de acidez, índice de saponificación, color y sólidos suspendidos) determinó que estos podrían ser utilizados como materia prima para la elaboración de jabón.
\end{abstract}

Palabras clave: aceite comestible usado; aceite rojo de palma; carbón activado; ozonización; peróxido de hidrógeno.

\begin{abstract}
:
Alternative processes for the clarification of used cooking oil (UCO) and the discoloration of red palm oil (RPO) were evaluated. The clarification of UCO consisted of two steps, sediment removal and bleaching. In the first step, three methods were tested: heating, washing with brine, sedimentation and filtration; sedimentation alone; and washing with brine, sedimentation and filtration. The third method allowed the highest removal of impurities. For the second step, three methods were proved: adsorption with activated carbon (AC); ozonation, and application of hydrogen peroxide. The best method was ozonation, with a dose of $0.1946 \mathrm{~mol} \mathrm{O}_{3} / \mathrm{L}$ and a posttreatment temperature of $60{ }^{\circ} \mathrm{C}$; a $24.39 \%$ discoloration was reached. Regarding RPO, the best treatment was the adsorption with activated carbon. The ratio of oil: AC that produced the best results was 25:1; a $90.48 \%$ color decrease was achieved. The analysis of the quality parameters measured in the treated oils (acid value, saponification value, color and suspended solids) determined that both could be used as raw material for manufacturing soap.
\end{abstract}

Keywords: used cooking oil; red palm oil; activated carbon; ozonation; hydrogen peroxide.

\footnotetext{
${ }^{1}$ Escuela Politécnica Nacional, Quito, Ecuador ( \{tania.parra, gonzalo.jacome, marco.sinche\} @epn.edu.ec ).

2 Universidad Tecnológica Equinoccial, Quito, Ecuador (freddy.marin@ute.edu.ec).
} 


\section{Introducción}

El aceite de cocina es un producto de consumo masivo, que se utiliza en la preparación de diversos alimentos. Sin embargo, una inadecuada disposición del aceite de cocina usado (ACU) significa, actualmente, un serio problema de contaminación ambiental; por ejemplo, se conoce que un litro de este residuo puede contaminar un millón de litros de agua (Restrepo, 2012). Cuando el aceite entra en contacto con el agua, se forma una película superficial que disminuye el intercambio de oxígeno e incrementa la mortalidad de los seres vivos del ecosistema acuático (Bureau Veritas, 2008).

La descarga doméstica de aceites y grasas, además, puede generar una acumulación de estos materiales en tuberías y demás instalaciones de fontanería, con su consecuente obstrucción y la reducción de su capacidad de almacenamiento y transporte (Husain et al., 2014; Metcalf \& Eddy Inc. et al., 2013). Sumado a lo anterior, la presencia de lípidos incrementa las tasas de corrosión, la necesidad de limpieza y mantenimiento, y el volumen de residuos sólidos que llegan a las plantas de tratamiento de aguas residuales (Arthur y Blanc, 2013). También, la grasa puede inhibir la actividad de los microorganismos utilizados en los tratamientos biológicos de efluentes, lo que conlleva una reducción de su efectividad (Aymong, 2007).

En la ciudad de Quito, se generan alrededor de $303 \mathrm{t}$ de ACU cada año, de las cuales solo el $10 \%$ es entregado a gestores ambientales (Secretaria de Ambiente del DMQ, 2011); el resto es desechado a alcantarillas o cuerpos de agua (INEC, 2013), a pesar que la Ordenanza Metropolitana No. 404 lo prohíbe. Los gestores destinan el ACU a la producción de alimentos para animales; sin embargo, esto puede ocasionar una acumulación de hidrocarburos y otros productos que generan daños en los tejidos biológicos, la aparición de enfermedades degenerativas y la disminución de la velocidad de crecimiento y de la conversión alimenticia en los animales que los ingieren (Delgado, 2004) y podrían acumularse en su carne y llegar eventualmente hasta el ser humano. Por ello, la Unión Europea ha prohibido esta práctica desde el 2002 (Cvengros y Cvengrosova, 2004). Es necesario buscar alternativas para la disposición del ACU que no generen potenciales problemas de salud ni contaminación ambiental.

EI ACU presenta un color marrón negruzco y tiene un sabor y olor característicos, además contiene ácidos grasos libres, triglicéridos, fosfolípidos, tocoferoles, esteroles, hidratos de carbono y otros compuestos, que resultan de reacciones termolíticas, oxidativas e hidrolíticas durante procesos como la fritura (Watt, 2013; Kulkarni y Dalai, 2006). Sin embargo, si este aceite es tratado para la remoción de sustancias indeseables, podría ser utilizado como materia prima para la elaboración de biodiésel, a través de un proceso de transesterificación, o de jabón, a través de un proceso de saponificación.

Varias investigaciones se han enfocado en el uso del ACU para la elaboración de biodiésel, principalmente debido a la reducción de costos de producción que esto representaría. Se han probado catalizadores ácidos, básicos y enzimáticos, a diferentes condiciones de operación; cada uno con sus propias ventajas y desventajas (Kulkarni y Dalai, 2006). La transesterificación catalizada por $\mathrm{NaOH}$ es, probablemente, la alternativa más estudiada, debido a su mayor velocidad de reacción; sin embargo, este proceso presenta un importante inconveniente asociado con la presencia de agua y ácidos grasos libres en el aceite, lo cual favorece a la reacción de saponificación, en lugar de la transesterificación (Enweremadu y Mbarawa, 2009; Math, Kumar y Chetty, 2010).

La elaboración de jabón es otra alternativa para una mejor disposición del ACU. En este caso, es de especial importancia remover los contaminantes que proporcionan coloración al aceite, puesto que se ha comprobado que, si el color no es eliminado en la materia prima, se manifiesta en el producto final (Watt, 2013). Debido a la cantidad de lípidos que se requeriría para la elaboración de jabón a una escala industrial, se podría utilizar, adicionalmente, el aceite rojo de palma (ARP), que es el producto de la extracción mecánica del fruto de palma africana (Elaeis guineensis). De manera similar, el ARP 
debe ser tratado previamente, con el fin de eliminar su pigmentación, la cual se debe principalmente a la presencia de carotenoides (Goh, Choo y Ong, 1985).

En el presente trabajo se evaluaron tres métodos alternativos de decoloración del ACU y del ARP, orientados hacia la denominada "producción más limpia", en la cual se busca eliminar, en lo posible, todo tipo de efluentes y desechos. En la metodología se describen las condiciones de operación estudiadas y los diseños experimentales utilizados para seleccionar el mejor método para cada tipo de aceite. Posteriormente se presentan los resultados referentes a los parámetros de calidad analizados en los aceites tratados y se discuten las causas por las cuales distintos métodos resultaron ser más efectivos para cada tipo de aceite. Por último, se analiza si los aceites tratados podrían ser empleados como materia prima para la elaboración de jabón.

\section{Materiales y Métodos}

\subsection{Materiales}

Se recolectaron muestras de $400 \mathrm{~mL}$ de $A C U$ en 50 locales de comida rápida y restaurantes del sector de La Mariscal (Quito, Ecuador) en botellas de vidrio, las cuales fueron mezcladas para obtener una muestra compuesta. El ARP fue obtenido de una empresa extractora ubicada en San Lorenzo, provincia de Esmeraldas, Ecuador.

\subsection{Determinación del Índice de Acidez, Índice de Saponificación y Contenido de Impurezas Insolubles presentes en el ACU y ARP}

La medición de los parámetros de calidad seleccionados para este estudio fue realizada de acuerdo con las normas INEN-ISO 660 (2013), INEN-ISO 3657 (2013) e INEN-ISO 663 (2013), correspondientes al índice de acidez, índice de saponificación y contenido de impurezas insolubles, respectivamente.

\subsection{Determinación del mejor proceso de eliminación de sedimentos presentes en el ACU}

Se compararon tres tipos de procesos. El primero consistió en un calentamiento a $90^{\circ} \mathrm{C}$; un lavado con salmuera al $5 \%$ de $\mathrm{NaCl}(\mathrm{p} / \mathrm{p})$ en relación $10: 1$, con agitación a 60 rpm por 30 min; sedimentación (7 h); y una filtración (Girgis, 2004). El segundo proceso fue solamente sedimentación y se probaron tiempos de 5, 6 y $7 \mathrm{~h}$. El tercero consistió en un lavado con salmuera y agitación a $60 \mathrm{rpm}$ por $30 \mathrm{~min}$, en el que se probaron relaciones de 10:1, 5:1 y 2,5;1; una sedimentación por $1 \mathrm{~h}$ y una filtración. Se realizaron tres repeticiones de cada tratamiento.

Después de la eliminación de sedimentos, se midieron el índice de acidez, índice de saponificación y contenido de impurezas insolubles y se realizó el balance de masa del proceso. Los datos fueron analizados en el programa Statgraphics Centurion XVI, mediante un ANOVA y una prueba de rangos múltiples por el método de Fisher (LSD).

\subsection{Determinación del mejor proceso de decoloración para cada tipo de aceite}

Se estudió la decoloración del ACU resultante del mejor proceso de eliminación de sedimentos (etapa anterior) y del ARP, a través de tres métodos: ozonización, adsorción con carbón activado $(\mathrm{CA})$ y adición de peróxido de hidrógeno.

Se llevaron a cabo pruebas preliminares con el fin de seleccionar las variables que tenían mayor influencia sobre la decoloración de ambos tipos de aceite, y sus niveles (Gutiérrez y De La Vara, 2012). Se seleccionaron las condiciones de operación de cada método que se probarían experimentalmente. Luego, se utilizaron diseños unifactoriales 
para determinar el mejor proceso de decoloración de cada aceite. Se realizaron tres repeticiones para cada tratamiento.

El proceso de ozonización se realizó con un ozonificador PHILAQUA, modelo BMT $802 \mathrm{M}$, con alimentación de oxígeno industrial, según el método descrito por Muñoz y Paredes (2014). Se trabajó con aceite a temperatura ambiente $\left(16^{\circ} \mathrm{C}\right)$ y aceite calentado a $20^{\circ} \mathrm{C}$ durante el proceso, debido a la influencia de este factor en la transferencia de masa en sistemas gas-líquido (Watt, 2013). Además, se probaron dos temperaturas postratamiento, 20 y $60 \stackrel{\circ}{\circ}$, debido a que estas podrían influir en la degradación del ozono residual. Se probaron dosis de $\mathrm{O}_{3}$ de 0,$0649 ; 0,1297$ y $0,1946 \mathrm{~mol} / \mathrm{L}$, las cuales se lograron al modificar los tiempos del proceso.

En la adsorción con CA, se utilizó carbón microporoso $(2 \mu \mathrm{m})$ de marca Panreac, en dos relaciones de aceite:CA (25:1 y 50:1). En la decoloración con peróxido de hidrógeno, se utilizó un reactivo de grado analítico, marca Mallinckrodt, en concentración 50\%, relación 1:1, con agitación a 80 rpm por 30 min (Bombón y Albuja, 2014).

La variable de respuesta, en todos los casos, fue el color de cada aceite luego de aplicar los diferentes tratamientos. Para ello, se midió la absorbancia a $540 \mathrm{~nm}$, en un espectrofotómetro UV-Vis, Hitachi, modelo U-1900. El blanco fue aceite de palma sin usar, puesto que permitió tener lecturas de absorbancia dentro del rango entre 0,0 y 2,0 (Sierra et al., 2010). Se empleó el aceite de la marca más utilizada en los establecimientos de producción de frituras, el cual fue identificado durante el muestreo.

Para medir el color del aceite rojo, se determinó la longitud de onda de máxima absorbancia mediante un barrido en un rango de 420 a $620 \mathrm{~nm}$, debido a los colores amarillo y rojo que presenta este aceite (Wannahari, Mariah y Nordin, 2012). Las lecturas de absorbancia de los aceites fueron realizadas por triplicado.

Finalmente, se determinaron los parámetros de calidad en los aceites resultantes de los mejores procesos de decoloración, puesto que son indicativos de la factibilidad de usarlos aceites en la industria jabonera (Hernández, Santamaría y Ríos, 2009).

\section{Resultados y Discusión}

\subsection{Parámetros de calidad en el aceite de cocina usado (ACU) y el aceite rojo de palma (ARP) antes de su tratamiento}

En la Tabla 1 se reportan los valores iniciales de índice de acidez, índice de saponificación y contenido de impurezas insolubles del ACU y del ARP. En el Ecuador no existe una norma que detalle los requisitos para que un aceite pueda ser utilizado como materia prima en la elaboración de productos de limpieza. Por tal motivo, en este estudio se consideraron algunos parámetros indicados en las normas INEN 2421:2009 y 2678:2013, referentes a los requerimientos para que el aceite rojo de palma y el aceite comestible reutilizado sean aptos para el consumo humano, respectivamente.

Ninguno de los aceites cumplió con los parámetros de calidad indicados en las normas, aunque el ARP tuvo mejores características, debido a que este aceite no ha sido sometido a procesos que generen un deterioro, al contrario de lo que sucede con el ACU.

\subsection{Mejor proceso de eliminación de sedimentos presentes en el ACU}

En los tres parámetros de calidad medidos, el tercer proceso presentó los mejores resultados, con un nivel de confianza de 95\%. El proceso seleccionado consistió en un lavado con salmuera al $5 \%$ de $\mathrm{NaCl}(\mathrm{p} / \mathrm{p})$ en una relación aceite:salmuera de 2,5:1 $(\mathrm{v} / \mathrm{v})$, durante $30 \mathrm{~min}$, a $40{ }^{\circ} \mathrm{C}$ y con agitación a $60 \mathrm{rpm}$, seguido de una sedimentación por $1 \mathrm{~h}$ y una filtración. Se consideró solo el $5 \%$ de $\mathrm{NaCl}$ para realizar la salmuera, ya que de acuerdo con la ley de Debye-Hückel, al encontrarse un electrolito fuerte en solución, sus iones se distribuyen de manera desigual, lo que provoca que cada ion negativo se rodee con un promedio mayor de iones positivos que de negativos, y viceversa (Jiménez, 2006). 
Tabla 1. Índice de acidez, índice de saponificación y contenido de impurezas insolubles del ACU y el ARP iniciales y valores de norma.

\begin{tabular}{|l|c|c|c|}
\hline & $\begin{array}{c}\text { Índice de acidez } \\
(\mathbf{m g} / \mathbf{g})\end{array}$ & $\begin{array}{c}\text { Índice de } \\
\text { saponificación } \\
(\mathbf{m g} / \mathbf{g})\end{array}$ & $\begin{array}{c}\text { Contenido de impurezas } \\
\text { insolubles (\%) }\end{array}$ \\
\hline ACU & $4,49 \pm 0,08$ & $217,07 \pm 0,70$ & $3,32 \pm 0,02$ \\
\hline ARP & $2,36 \pm 0,04$ & $190,84 \pm 0,49$ & $2,07 \pm 0,02$ \\
\hline Valor norma $^{* *}$ & $\begin{array}{c}\text { Máx. 3,0 (ACU) } \\
\text { Máx. 0,3 (ARP) }\end{array}$ & 189 a 199 (ARP) & $0,05(\mathrm{ARP})$ \\
\hline
\end{tabular}

*Como ácido oleico

**(INEN 2421:2009 e INEN 2678:2013)

$\bar{x} \pm \sigma(n=3)$

Los mejores resultados alcanzados se deberían a que el lavado con salmuera mejoró el proceso de floculación de impurezas, gracias a que la sal se disocia al momento de entrar en contacto con el agua y sus iones neutralizan la carga sobre los sedimentos presentes en el aceite, lo que ayuda a que estos se agreguen y sedimenten (Kotz, Treichel y Weaver, 2006). Esto concuerda con lo expuesto por Sierra, Yañez y Cruz (2006), quienes enunciaron que cuando las capas de las partículas de los sedimentos entran en contacto con la solución de $\mathrm{NaCl}$, absorben iones positivos y negativos, lo que resulta en una disminución de las fuerzas de repulsión y un incremento en la coalescencia de la fase dispersa (aceite).

EI ACU resultante del mejor tratamiento de eliminación de impurezas presentó un índice de acidez de 2,09, un índice de saponificación de 185,14 y un contenido de impurezas insolubles de $1,03 \%$. Solamente el tercer parámetro tuvo un valor fuera de las normas tomadas como referencia.

\subsection{Mejor proceso de decoloración para el ACU}

\subsubsection{Condiciones de operación para el proceso de ozonización}

En la Figura 1, se muestran los gráficos de absorbancia en función del tiempo, para los cuatro tratamientos del proceso de ozonización del ACU. Se midió la absorbancia por 13 días, con el fin de determinar el tiempo en el cual termina de reaccionar el ozono. La dosis de ozono utilizada $(0,1946 \mathrm{~mol} / \mathrm{L})$ fue seleccionada mediante ensayos preliminares, en los que se observó que mientras menor era la dosis de ozono, mayor tendía a ser el tamaño de las burbujas, debido a que un menor caudal favorece el flujo laminar y la coalescencia de las gotas. Al formarse burbujas más grandes, disminuye el área interfacial y como consecuencia, se reduce la transferencia de masa entre el ozono y el ACU.

Se puede notar que la absorbancia del aceite continúa disminuyendo hasta aproximadamente 10 días después de la ozonización, en los aceites sin calentamiento posterior. Esto se podría deber a que las burbujas que se generaron en el proceso tuvieron un largo tiempo de residencia, especialmente las de mayor tamaño, debido a la alta viscosidad del medio (Xiong et al., 2003). Al romperse dichas burbujas con el paso de los días, continuaron generando reacciones de decoloración. Además, en el estudio de Cirlini et al. (2012), se analizó la estabilidad de los ozónidos en el aceite de girasol y se determinó que después de $16 \mathrm{~h}$ de reacción, los ozónidos secundarios aún se encontraban presentes en el aceite; estos podrían seguir reaccionando y produciendo la destrucción de moléculas que proporcionan color al ACU.

Además, se observa que al tiempo cero, los valores de absorbancia son diferentes. Esto concuerda con lo antes expuesto, debido a que cuando el ozono tiene mayor área de contacto con el aceite, puede reaccionar más eficientemente y degradar las moléculas con color. Cabe indicar que, al momento de realizar la medición de la 
absorbancia, las burbujas de ozono que seguían presentes en el aceite tratado pudieron interferir en la trayectoria del haz de luz. Esto puede explicar la tendencia sigmoidal que se observa en las curvas en mención (Rubinson y Rubinson, 2001).

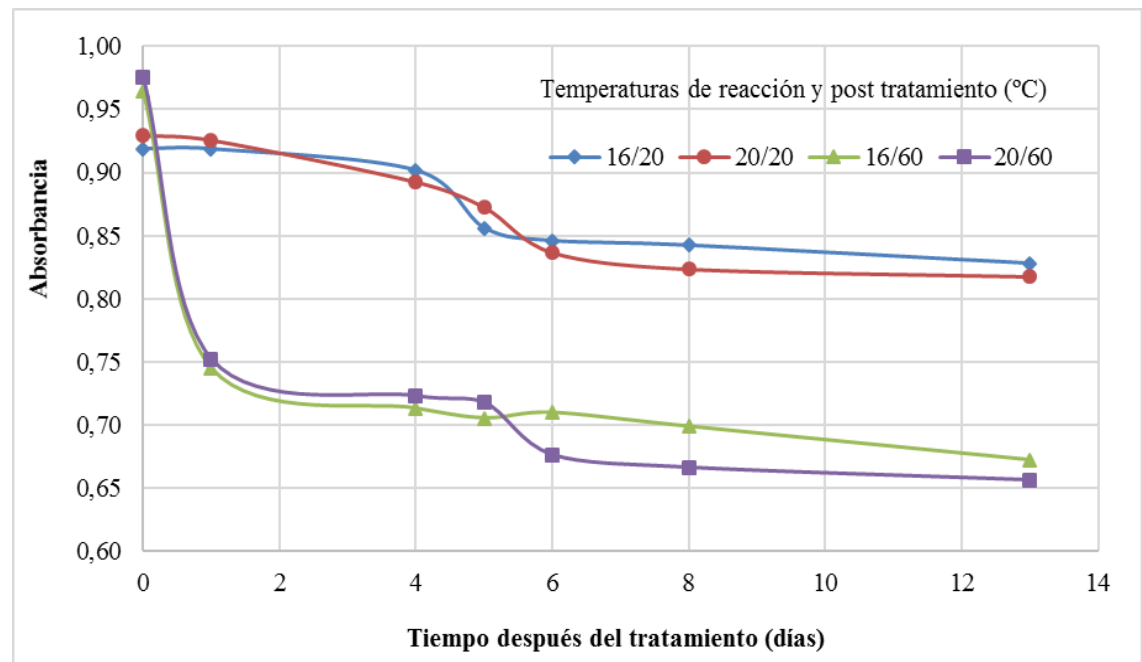

Figura 1. Absorbancia de las muestras de ACU tratadas por ozonización, a diferentes temperaturas de reacción $\left(16 \circ 20^{\circ} \mathrm{C}\right)$ y con o sin calentamiento postratamiento $\left(20 \circ 60^{\circ} \mathrm{C}\right)$

Al comparar los valores de absorbancia obtenidos cuando se variaron las temperaturas de reacción y postratamiento, se encontró que los dos mejores resultados correspondieron a los tratamientos que tuvieron un calentamiento posterior. Las diferencias se podrían atribuir a la disminución de la viscosidad que se produce cuando el aceite se calienta, lo que puede facilitar la reacción. De acuerdo con Xiong et al. (2003), una mayor viscosidad de la fase líquida se relaciona con un menor coeficiente de transferencia de masa del gas en el líquido.

En la Figura 1, además, se puede observar que el tiempo que el aceite requeriría ser almacenado luego del tratamiento y previo a su uso, puede ser menor cuando se aplica un calentamiento posterior, con un valor alrededor de 6 días hasta que alcance una absorbancia estable, que indicaría que el ozono remanente terminó de descomponerse.

Los dos viales del lado izquierdo de la Figura 2 corresponden a los casos en los que no existió un calentamiento postratamiento, y presentan una coloración más oscura. El cuarto vial (lado derecho) presentó una coloración muy similar a la del aceite vegetal no usado, lo cual es un indicativo que se alcanzaron los resultados esperados. Los porcentajes de decoloración en cada caso, en el mismo orden, fueron: 4,61 $\pm 5,01 ; 5,80 \pm$ 2,$37 ; 22,58 \pm 1,58$ y $24,39 \pm 2,35 \%$, con respecto al ACU obtenido tras la eliminación de sedimentos descrita en la sección 3.2 .

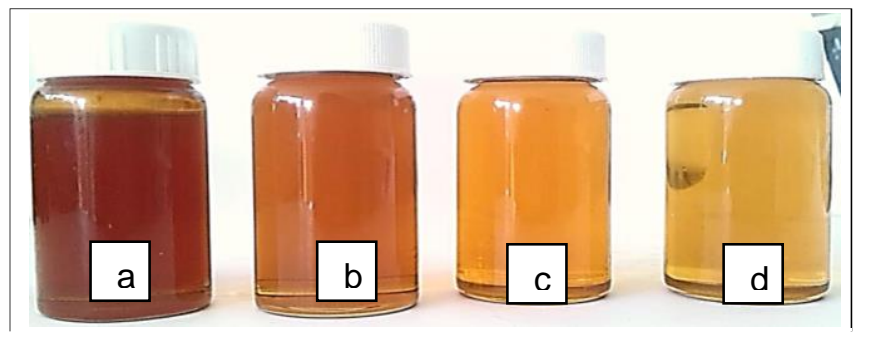

Figura 2. Comparación del color en el ACU luego de la ozonización, a diferentes temperaturas de reacción y postratamiento: a) 16 y $22^{\circ} \mathrm{C}$, b) 20 y $20^{\circ} \mathrm{C}$, c) 16 y $60^{\circ} \mathrm{C}$, d) 20 y $60^{\circ} \mathrm{C}$

El análisis estadístico de la Figura 3 determinó que la temperatura durante la ozonización no influyó significativamente $(p>0,05)$ sobre el color alcanzado, pero la temperatura postratamiento sí. En consecuencia, se determinó que las mejores 
condiciones para la ozonización del ACU fueron a una temperatura de reacción de $16 \stackrel{\circ}{ } \mathrm{C}$ (ambiente), una dosis de ozono de $0,1946 \mathrm{~mol} / \mathrm{L}$ y un calentamiento postratamiento de 60 ${ }^{\circ} \mathrm{C}$. El uso de una temperatura ambiente durante la reacción representa un menor gasto energético, lo cual se reflejaría como un ahorro económico.

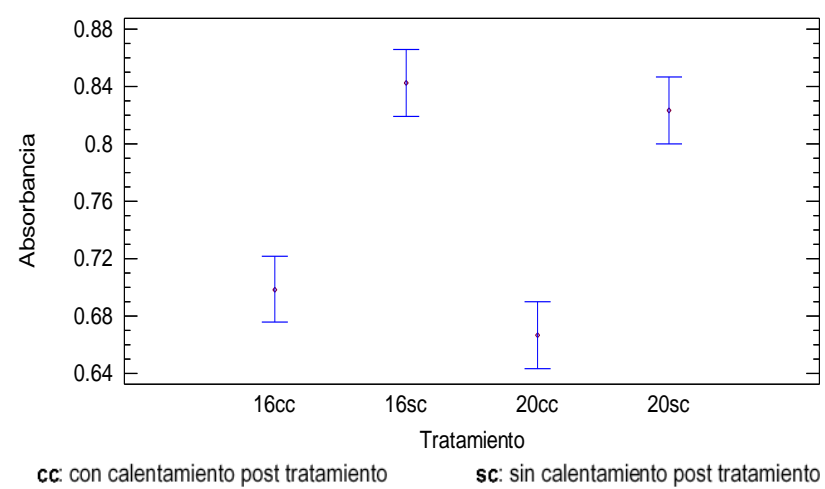

Figura 3. Gráfico de medias de la absorbancia del ACU luego de la ozonización a diferentes temperaturas de reacción, con y sin calentamiento posterior

\subsubsection{Comparación entre los tres procesos de decoloración del ACU}

Para determinar el mejor proceso de decoloración del ACU, se compararon los valores de absorbancia de las muestras de aceite tratado con ozono, peróxido de hidrógeno o carbón activado. Los resultados se muestran en la Tabla 2 y la Figura 4.

Tabla 2. Valores de absorbancia a $540 \mathrm{~nm}$ luego de tratar el ACU con los tres procesos de decoloración.

\begin{tabular}{|c|c|c|c|c|}
\hline & Blanco & Ozonización & $\begin{array}{c}\text { Aplicación de } \\
\mathbf{H}_{2} \mathbf{O}_{2}\end{array}$ & $\begin{array}{c}\text { Adsorción con } \\
\text { carbón activado }\end{array}$ \\
\hline Absorbancia & $0,868 \pm 0,001$ & $0,660 \pm 0,002^{\mathrm{c}}$ & $0,740 \pm 0,002^{\mathrm{b}}$ & $1,200 \pm 0,003^{\mathrm{a}}$ \\
\hline \% Decoloración & - & 24,39 & 14,63 & $-38,10$ \\
\hline
\end{tabular}

$\bar{x} \pm \sigma(\mathrm{n}=3)$

a,b,c: Letras distintas representan diferencias significativas (Fisher, 95\% de confianza)

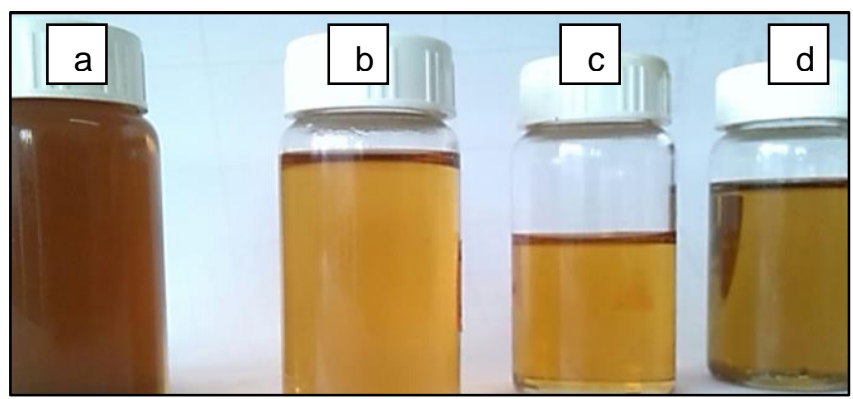

Figura 4. Muestras de ACU: a) sin tratar, b) tratado por ozonización, c) decolorado con peróxido de hidrógeno y c) tratado por adsorción con carbón activado

El análisis estadístico determinó que el proceso de ozonización permitió obtener los mejores resultados $(p<0,05)$. Esto se debería a que en el ACU se pueden encontrar productos del proceso de fritura que presentan en su estructura dobles enlaces carbonocarbono, los cuales pueden romperse por la acción del ozono, según el mecanismo descrito por Criegge (1975), y generar moléculas de menor tamaño. Nandini y Sivasakthivel (2014) concluyeron que el ozono en medios oleosos podría tener ventajas 
sobre los medios gaseosos o acuosos, puesto que permanecería durante un período más largo y generaría una mayor cantidad de reacciones de ozonólisis

Además, el porcentaje de decoloración correspondiente a la adsorción con CA, en contraste con los otros métodos, presentó un valor negativo. Esto se explica porque parte del carbón añadido se quedó suspendido en el aceite y no pudo ser eliminado por filtración. Según Gibon, De Greyt y Kellens (2007), en los aceites sometidos a procesos de oxidación, como la fritura, se pueden desarrollar compuestos coloreados de tipo quinónico y, también, los pigmentos propios del aceite se estabilizan, lo cual dificulta su eliminación por adsorción física.

\subsection{Mejor proceso de blanqueamiento para el ARP}

Para seleccionar el mejor proceso de blanqueamiento del ARP, se compararon los resultados del color obtenido al aplicar tres tipos de tratamiento: adsorción con carbón activado, ozonización y decoloración con peróxido de hidrógeno. Como primer paso, se determinaron las mejores condiciones de operación del proceso de adsorción con carbón activado (CA), puesto que en pruebas preliminares fue el que mostró los mejores resultados. Se observó que, a las mismas condiciones de agitación, el carbón microporoso se mantuvo en suspensión por mayor tiempo que el carbón macroporoso; además, sus partículas pueden dispersarse más rápidamente en el aceite a temperaturas más altas, lo que favorece al proceso de adsorción (Brooks, Berbesi y Hodgson, 2013). Por tanto, se seleccionó el CA microporoso con tamaño de poro de $2 \mu \mathrm{m}$.

\subsubsection{Determinación de las mejores condiciones de operación del proceso de adsorción con carbón activado para el ARP}

En la Figura 5, se presenta el gráfico de medias de la absorbancia del ARP luego de su tratamiento mediante adsorción con CA. Se consideraron dos variables de diseño, con dos niveles cada una: temperatura de adsorción $\left(110\right.$ y $\left.140{ }^{\circ} \mathrm{C}\right)$ y relación aceite:CA (25:1 y 50:1). El tratamiento que presentó los menores valores de absorbancia fue aquel en el que se aplicó como temperatura de adsorción $140^{\circ} \mathrm{C}$ y la relación aceite:CA de 25:1. La temperatura y la relación aceite:CA influyeron significativamente en el proceso de decoloración del ARP. Se obtuvieron mejores resultados cuando se incrementó la temperatura, porque en ese caso, la viscosidad del aceite disminuye; como resultado, la resistencia al flujo es menor y se incrementa la capacidad del ARP de mantener una partícula en suspensión (Brooks et al., 2013). Al mantenerse suspendido, el CA puede adsorber de mejor manera los pigmentos que dan la coloración al aceite.

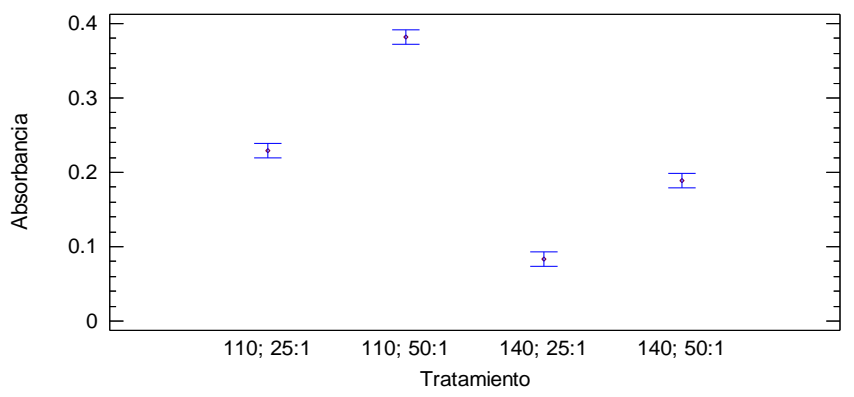

Figura 5. Gráfico de medias de la absorbancia del ARP tratado por adsorción con carbón activado a diferentes temperaturas y relaciones aceite:CA

Al analizar el efecto de la relación aceite:CA, se encontró que la proporción que generó una mayor remoción de color fue la de 25:1. Esto puede deberse a que se tuvo una mayor cantidad de partículas de CA dispersas en el aceite y, por ende, una mayor 
superficie de contacto para la adsorción de los pigmentos. Probablemente, cuando la relación fue de 50:1, las partículas de carbón llegaron a saturarse.

Nandini y Sivasakthivel (2014) estudiaron la decoloración de aceite de girasol usado con CA y observaron una variación directamente proporcional de la eficiencia de blanqueo con el tiempo de contacto y con la masa del material adsorbente. Al tener una mayor cantidad de CA, el área de contacto en la que los pigmentos vegetales son adsorbidos también es mayor; además, con el tiempo, una mayor cantidad de aceite entra en contacto con el CA y los pigmentos quedan retenidos.

\subsubsection{Comparación entre los tres procesos de decoloración para el ARP}

Los resultados del color del ARP tratado mediante los tres procesos de blanqueamiento propuestos: ozonización, adición de peróxido de hidrógeno y adsorción con carbón activado bajo las condiciones de operación determinadas en la sección 3.4.1.se presentan en la Tabla 3.

Tabla 3. Valores de adsorbancia a $540 \mathrm{~nm}$ luego de tratar el ARP con los tres procesos de decoloración.

\begin{tabular}{|c|c|c|c|c|}
\hline & $\begin{array}{c}\text { Aceite sin } \\
\text { tratar }\end{array}$ & Ozonización & $\begin{array}{c}\text { Aplicación de } \\
\mathbf{H}_{2} \mathbf{O}_{2}\end{array}$ & $\begin{array}{c}\text { Adsorción con } \\
\text { carbón activado }\end{array}$ \\
\hline Adsorbancia & $0,949 \pm 0,002$ & $0,856 \pm 0,005^{\mathrm{a}}$ & $0,806 \pm 0,008^{\mathrm{b}}$ & $0,083 \pm 0,002^{\mathrm{c}}$ \\
\hline \% Decoloración & - & 9,80 & 15,10 & 91,29 \\
\hline
\end{tabular}

$\bar{x} \pm \sigma(n=3)$

Letras distintas representan diferencias significativas (Fisher, 95\% de confianza)

El mejor proceso de decoloración para el ARP fue la adsorción con CA, el cual presentó una disminución del color del 91,29\%, con respecto al aceite sin tratar. Otros estudios acerca del blanqueamiento de ARP han obtenido resultados semejantes. Maskan y Bağcı (2003) determinaron que el tratamiento de ARP con CA disminuyó su color y su viscosidad. Kheang, Subari y Kadir (2011) también encontraron que los mejores resultados de decoloración de oleína de palma correspondieron al blanqueamiento con CA.

En la Figura 6 se presentan las muestras de ARP, luego de aplicar los tres procesos de blanqueamiento, junto a la muestra sin tratamientos. Se puede notar que el mejor proceso de blanqueamiento fue la adsorción con CA. En contraste con lo ocurrido con el ACU, la ozonización generó la menor remoción de color en el ARP; esto podría deberse a la presencia de agua en el aceite, puesto que, según el mecanismo de ozonólisis propuesto por Criegee (1975), el agua favorece la formación de hidroperóxidos y la consecuente ruptura de los enlaces dobles carbono-carbono. En el ACU existe agua, la cual se transfiere desde los alimentos durante el proceso de fritura, pero en el ARP no.

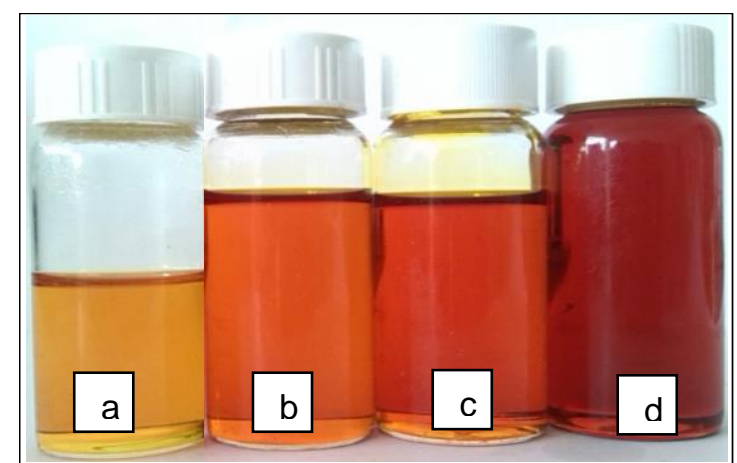

Figura 6. Muestras de ARP luego de la decoloración con: a) carbón activado, b) peróxido de hidrógeno, c) ozono, y d) aceite sin tratar 
En resumen, el mejor tratamiento de decoloración para el ARP no fue el mismo que para el ACU. Una razón es la diferencia del tipo de compuestos coloreados de cada aceite; los carotenoides le dan un color rojizo al ARP, mientras que compuestos quinónicos generados durante el proceso de fritura le dan al ACU una coloración marrón oscura. La adsorción con carbón activado fue eficiente para remover los carotenoides, mientras que la ozonización presentó mejores resultados en la degradación de los productos de la fritura.

\section{Conclusiones y recomendaciones}

El ARP y el ACU, antes de los tratamientos, presentaron índices de acidez, índices de saponificación y contenidos de impurezas insolubles superiores los valores estipulados por las normas INEN 2421:2009 y 2678:2013.

El mejor proceso de eliminación de sedimentos para el ACU (lavado con salmuera, sedimentación y filtración) presentó porcentajes de disminución en los parámetros de calidad de: $53,45 \%$ en el índice de acidez, $14,71 \%$ en el índice de saponificación y $68,98 \%$ en el contenido de impurezas insolubles. La ozonólisis con una dosis de 0,1946 $\mathrm{mol} \mathrm{O}_{3} / \mathrm{L}$, temperatura de reacción ambiente $\left(16^{\circ} \mathrm{C}\right)$ y calentamiento postratamiento de $60 \stackrel{\circ}{\circ}$, fue el mejor proceso de blanqueamiento, con un $24,39 \%$ de decoloración.

El mejor proceso de blanqueamiento del ARP fue la adsorción con CA con tamaño de poro $2 \mu \mathrm{m}$, a $140^{\circ} \mathrm{C}$, con una relación aceite:CA de $25: 1$ y agitación a $60 \mathrm{rpm}$ por 15 min. La disminución de color fue del 90,48\%. Además, la remoción del carbón al final del tratamiento no generó inconvenientes, a diferencia de lo ocurrido con el ACU; esto podría deberse a factores como la viscosidad o el tipo de contaminantes de cada aceite.

Por los resultados alcanzados, se estima que los aceites tratados serían aptos para ser utilizados como materia prima en la elaboración de jabón, pues los requisitos serían menos rigurosos en comparación con los requisitos para su uso en la alimentación humana. Se recomienda estudiar las condiciones del proceso de saponificación a partir de estos materiales, que permitan obtener el mayor rendimiento y calidad de jabón.

\section{Bibliografía}

Arthur, S. y Blanc, J. (2013). Management and Recovery of FOG (fats, oils and greases), CREW project CD2013/6. Recuperado de: http://www.crew.ac.uk/sites/default/ files/sites/default/files/publication/CREW_FOG.pdf (accedido el 25/05/2018).

Bombón, N. y Albuja, M. (2014). Diseño de una planta de saponificación para el aprovechamiento del aceite vegetal de desecho. Revista Politécnica, 34(1), 22-31.

Brooks, D. Berbesi, R. y Hodgson A. (2013). Optimization of the bleaching process. Recuperado de http://lipidlibrary.aocs.org/processing/bleaching/index.htm (accedido el 17/03/2015).

Bureau Veritas. (2008). Manual para la formación en Medio Ambiente. Recuperado de https://books.google.com.ec/books/about/Manual_para_la_formaci\%C3\%B3n_en_m edio_ambie.html?id=J7rMDpW49ZQC\&redir_esc=y (accedido el 15/01/2015).

Cirlini, M., Caligiani, A., Palla, G., De Ascentiis, A., y Tortini, P. (2012). Stability studies of ozonized sunflower oil and enriched cosmetics with a dedicated peroxide value determination. Ozone: Science \& Engineering, 34(4), p. 293-299. http://dx.doi.org/ 10.1080/01919512.2012.692992.

Criegee, R. (1975), Mechanism of Ozonolysis. Angew. Chem. Int. Ed. Engl., 14, p. 745752. http://dx.doi.org/10.1002/anie.197507451.

Cvengros, J. y Cvengrosova, Z. (2004). Used frying oils and fats and their utilization in the production of methyl esters of higher fatty acids. Biomass Bioenergy, 27, 173-181.

Delgado, W. (2004). ¿Por qué se enrancian las grasas y aceites? Revista Palmas 25(2), 35-43. Recuperado de http://publicaciones.fedepalma.org/index.php/palmas/ article/view/990 (accedido el 23/10/2014). 
Enweremadu, C.C. y Mbarawa, M.M. (2009). Technical aspects of production and analysis of biodiesel from used cooking oil-A review. Renewable and Sustainable Energy Reviews 13, p. 2205-2224. http://dx.doi.org/10.1016/j.rser.2009.06.007.

Gibon, V., De Greyt, W., y Kellens, M. (2007). Palm oil refining. European journal of lipid science and technology, 109(4), 315-335. http://dx.doi.org/10.1002/ejlt.200600307.

Girgis, A. Y. (2004). The utilization of discarded oil from potato chip factories in toilet soap making. Grasas y Aceites, 55(3), 264-272. Recuperado de http://grasasyaceites. revistas.csic.es/index.php/grasasyaceites/article/viewArticle/175 (accedido el 15/11/2014).

Goh, S.H., Choo Y.M. y Ong A.S.H. (1985). Minor constituents of palm oil. J. Am. Oil Chem. Soc.; 62:237-40.

Gutiérrez, H. y De La Vara, R. (2012). Análisis y diseño de experimentos (3a. ed.). México D.F.: McGrawHill.

Hernández, P. N. B., Santamaría, J. R. A. y Ríos, L. A. (2009). Biodiesel: Producción, calidad y caracterización. Recuperado de https://books.google.com.ec/books?id=yia ApKhNqRYC\&printsec=frontcover $\# v=$ onepage\&q\&f=false (accedido el 20/06/ 2015).

Husain, I. A. F., Alkhatib, M. F., Jammi, M. S., Mirghani, M. E. S., Zainudin, Z. B., y Hoda, A. (2014). Problems, Control, and Treatment of Fat, Oil, and Grease (FOG): A Review. J. Oleo Sci. 63, (8), p. 747-752. http://dx.doi.org/10.5650/jos.ess13182.

INEC. (2013). Módulo de Información Ambiental en Hogares ENEMDU. Recuperado de http://www.ecuadorencifras.gob.ec/documentos/web-inec/Encuestas_Ambientales/ Hogares2013/201401_EnemduAmbientePresentacion.pdf (accedido el 15/01/2014).

INEN-ISO 3657. (2013). Aceites y grasas de origen animal y vegetal: Determinación del contenido de impurezas solubles. (1a. ed.) Quito, Ecuador.

INEN-ISO 660. (2013). Aceites y grasas de origen animal y vegetal: Determinación del índice de acidez. (1a. ed.) Quito, Ecuador.

INEN-ISO 663. (2013). Aceites y grasas de origen animal y vegetal: Determinación del índice de saponificación. (1a. ed.) Quito, Ecuador.

Jiménez, M. C. (2006). Química física para ingenieros químicos. Servicio de publicación de la Universidad Politécnica de Valencia. ISBN: 9788497059466.

Kheang, L. S., Subari, F. y Kadir, S. A. S. A. (2011). Pre-treatment of palm olein-derived used frying oil as a feedstock for non-food applications. Journal of Oil Palm Research, 23, 1185-1192. Recuperado de http://www.researchgate.net/profile/Soh _Kheang_Loh/publication/260639918_pre-treatment_of_palm_olein-derived_used frying_oil_as_a_feedstock_for_non-food_applications/links/00 $\bar{b} 49539164$ a11255c00 0000.pdf (accedido el 30/03/2015).

Kotz, J., Treichel, P. y Weaver, G. (2006). Chemistry and chemical reactivity. Cengage Learning. Recuperado de https://books.google.com.ec/books/about/Chemistry_and Chemical_Reactivity.html?id=4vL3SjWjEcQC\&redir_esc=y (accedido el 18/03/ 2015).

Maskan, M. y Bağcl, H. (2003). Effect of different adsorbents on purification of used sunflower seed oil utilized for frying. European Food Research and Technology, 217(3), p. 215-218. http://dx.doi.org/10.1007/s00217-003-0731-2.

Math, M. C., Kumar, S. P. y Chetty, S. V. (2010). Technologies for biodiesel production from used cooking oil - A review. Energy for Sustainable Development 14, p. 339345. http://dx.doi.org/10.1016/j.esd.2010.08.001

Metcalf \& Eddy, Inc., Tchobanoglous, G., Burton, F. y Stensel, H. D. (2013). Wastewater Engineering: Treatment and resource Recovery (5a. ed.). McGraw-Hill. ISBN: 9780073401188.

Muñoz, F. y Paredes, A. (2014). Descontaminación de fenoles en el efluente de una refinería ecuatoriana, mediante el uso de ozono y combinaciones con peróxido de hidrógeno. Revista Politécnica, 34(1), 16-21.

Nandini, N. y Sivasakthivel. S. (2014). Bleaching of sunflower waste oil by absorption on 
activated carbon and improved by ozonisation. AIJRSTEM, 7(1), 35-39. Recuperado de http://iasir.net/AIJRSTEMpapers/AIJRSTEM14-522.pdf (accedido el 17/06/2015).

Restrepo, J. E. (2012). El desarrollo sostenible y el reciclaje del aceite usado de cocina a la luz de la jurisprudencia y el ordenamiento jurídico colombiano. Producción Más Limpia, 7(1), 109-122. Recuperado de http://www.scielo.org.co/pdf/pml/v7n1/ v7n1a11.pdf (accedido el 17/01/2015).

Rubinson, K. A. y Rubinson, J. F. (2001). Análisis instrumental. (1a. ed.). Madrid, España: Pearson Education.

Secretaría de Ambiente del Distrito Metropolitano de Quito. (2011). Residuos Peligrosos. Recuperado de la base de datos de la Secretaría de Ambiente del DMQ. (accedido el 18/01/2015).

Sierra, G., Yañez, E. E. y Cruz, C. (2006). Estudio de la factibilidad técnica sobre la decantación independiente de los recuperados de centrífuga en el proceso de extracción de aceite de palma. Revista Palmas, 27(2), 23-33. Recuperado de http://publicaciones.fedepalma.org/index.php/palmas/article/view/1176/1176 (accedido el 25/03/2015).

Sierra, I., Gómez-Ruiz, S., Pérez-Quintanilla, D. y Morante, S. (2010). Análisis instrumental. Algunas herramientas de enseñanza-aprendizaje adaptadas al Espacio Europeo de Educación Superior. Recuperado de https://books.google.com.ec/books?isbn= 8499699073 (accedido el 21/03/2015).

Wannahari, R., Mariah, F. y Nordin, M. (2012). The Recovery of Used Palm Cooking Oil Using Bagasse as Adsorbent. Am. J. Eng. \& Applied Sci. 5(1), 59-62. Recuperado de http://thescipub.com/PDF/ajeass p.2012.59.62.pdf (accedido el 25/10/2014).

Watt, A. (2013). The Art of Soap-Making. Recuperado de http://www. forgottenbooks.com/readbook_text/The_Art_of_Soap-Making1000849255/219 (accedido el 25/01/2015).

Xiong, R. H., Wang, S. C., Xu, S. C. y Wang, J. X. (2003). Experimental investigation of mass transfer in high viscosity media. International communications in Heat and Mass Transfer, 30(6), 817-824. http://dx.doi.org/10.1016/S0735-1933(03)00129. 